\title{
AVALIAÇÃO DA REGENERAÇÃO DOS MEIOS FILTRANTES APÓS DUZENTOS CICLOS DE FILTRAÇÃO POR PULSO DE AR REVERSO
}

\author{
S.S.R.CIRQUEIRA ${ }^{1}$, E.H. TANABE ${ }^{2}$; M.L.AGUIAR ${ }^{1}$ \\ ${ }^{1}$ Universidade Federal de São Carlos, Departamento de Engenharia Química \\ ${ }^{2}$ Universidade Federal de Santa Maria, Departamento de Engenharia Química \\ E-mail para contato: samirysara@gmail.com
}

\begin{abstract}
RESUMO - O objetivo do trabalho foi avaliar a regeneração dos meios filtrantes a duzentos ciclos de filtração e limpeza por pulso de ar reverso. Os ensaios de filtração foram realizados mantendo-se a velocidade de filtração constante a $4 \mathrm{~cm} / \mathrm{s}$, com queda de pressão máxima de $200 \mathrm{~Pa}$ e tempo de pulso de $200 \mathrm{~ms}$, a cada limpeza do tecido. O material particulado utilizado foi o concentrado fosfático de diâmetro médio 9,63 um e os filtros foram de acrílico e de polipropileno. Os parâmetros obtidos experimentalmente foram: queda de pressão residual, massa retida, fração removida, resistências específicas e porosidade da torta formada. Os resultados mostraram que apesar da massa retida e da queda de pressão residual serem maiores, as tortas de filtração apresentaram maior porosidade e, portanto menor resistência específica para os filtros avaliados.
\end{abstract}

\section{INTRODUÇÃO}

Os impactos ambientais causados pela poluição do ar industrial começaram a surgir com o fortalecimento do setor industrial e, a partir disso, a questão ambiental baseada na conservação, solidificou-se em todo o mundo, afetando a ciência e a tecnologia, nas últimas décadas. Consequentemente, a filtração por pulso de ar reverso tornou-se a escolha preferida em todo o mundo, como uma das principais operações na limpeza de correntes gasosas (Mukhopadhyay e Choudhary, 2013).

A operação de filtração por pulso de ar reverso é amplamente utilizada para controlar a emissão de partículas e para recuperar partículas no setor industrial, combinando o controle ambiental e o reaproveitamento de material particulado, sem perder a eficiência de coleta, em um processo contínuo de regeneração do meio filtrante, não apresentando a necessidade de interromper a filtração (Ginestet e Pugnet, 2012; Zhou et al., 2012).

Durante a filtração, o fluido contendo o material particulado passa por meio de um coletor poroso e permeável e, neste meio filtrante, uma camada de pó é depositada em sua superfície, formando a torta de filtração e resultando em um aumento linear da queda de pressão no filtro (Simon et al., 2010). Nesta etapa, os filtros devem ser periodicamente regenerados por meio da fase de limpeza utilizando pulso de ar reverso, para prevenir o alto consumo de energia. Por isso, o 


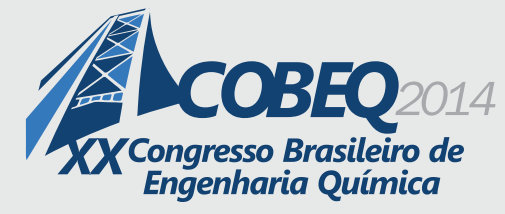

19 a 22 de outubro de 2014

Florianópolis/SC

desprendimento da torta de filtração é indispensável, uma vez que, o pulso de ar reverso fornece força necessária para separar a torta do meio filtrante. Nesta técnica de limpeza, normalmente, utilizam-se, feltros como meio filtrante e possibilita-se maior relação ar-tecido por meio da limpeza periódica dos filtros. Assim, o sucesso da operação de limpeza depende do meio filtrante, da porosidade da torta, da resistência específica da torta de filtração, da distribuição do material particulado por unidade de área do meio filtrante e da velocidade superficial de filtração (Saleem et al. 2011)

Portanto, a fase de limpeza por pulso de ar reverso consiste em remover as partículas do filtro, por um pulso à alta pressão, em um curto período de tempo e, consequentemente, o processo de filtração continua regularmente. Assim, nesta etapa a queda de pressão diminui para um valor inferior, conhecido como a queda de pressão residual (Allen et al., 1999). Entretanto, esta queda de pressão residual aumenta lentamente com o número de ciclos filtração, tendendo a um valor limite, em que as condições de estado estacionário são alcançadas e ocorre o desgaste do filtro (Simon et al., 2010). Allen et al. (1999) tentaram melhorar a compreensão do mecanismos de remoção da torta formada no filtro. Entretanto, Simon et al. (2007) observaram que a medida que o número de ciclos aumentava, a queda de pressão residual passava a ter um acréscimo. Mais recentemente, Park et al. (2012) investigaram a relação entre a penetração das partículas no meio filtrante e a queda de pressão neste meio. Diante deste contexto, este trabalho teve por objetivo avaliar a regeneração dos meio filtrantes submetidos a duzentos ciclos de filtração, utilizando a técnica de limpeza, por pulso de ar reverso.

\section{MATERIAIS E MÉTODOS}

\subsection{Material particulado}

O material particulado utilizado foi o concentrado fosfático, fornecido pela Fosfértil S.A, cuja massa específica corresponde a 3,03 g/ $\mathrm{cm}^{3}$ obtida por Picnometria a gás Hélio. Neste material foram detectadas partículas compreendidas em uma ampla faixa de diâmetro, com o diâmetro médio volumétrico de 9,63 $\mu \mathrm{m}$, obtida pelo equipamento Malvern Mastersizer Microplus. Ambas as análises foram realizadas no Laboratório de Controle Ambiental do DEQ/UFSCar.

\subsection{Meios filtrantes}

Os meios filtrantes utilizados para avaliar a regeneração após duzentos ciclos de filtração foram os feltros de polipropileno e de acrílico. Na Figura 1 são mostradas as imagens obtidas da superfície desses feltros em um Microscópio Eletrônico de Varredura (MEV). Na Tabela 1 são apresentadas as gramaturas desses meios filtrantes fornecidas pelo fabricante, os resultados das permeabilidades obtidas experimentalmente e os resultados do diâmetro médio das fibras determinados a partir da Figura 1 e pelo programa Image Pro Plus 7.0. 


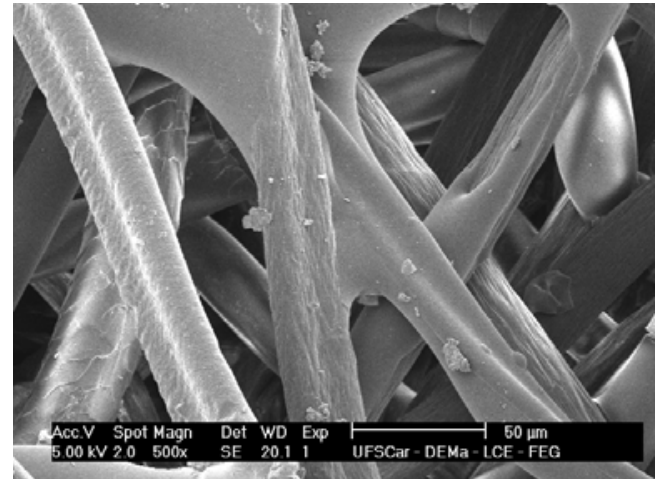

(a)

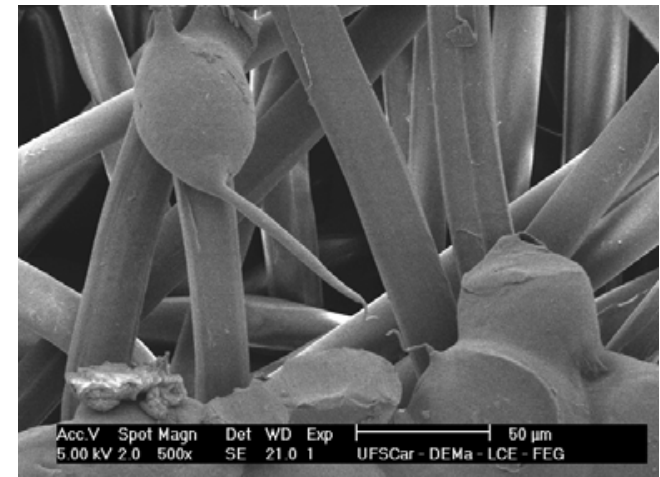

(b)

Figura 1 - Fotografia da superfície do feltro de (a) acrílico e de (b) polipropileno gerada no MEV.

Tabela 1 - Características dos meios filtrantes.

\begin{tabular}{c|c|c}
\hline Meio filtrante & Características do meio filtrante & Valores obtidos \\
\hline \multirow{3}{*}{ Polipropileno } & Diâmetro médio das fibras & $19,35 \mu \mathrm{m}( \pm 2,49)$ \\
\cline { 2 - 3 } & Gramatura $\left(\mathrm{g} / \mathrm{m}^{2}\right)$ & 600 \\
\cline { 2 - 3 } & Permeabilidade $\left(\mathrm{m}^{2}\right)$ & $9,7 \times 10^{-8}$ \\
\hline \multirow{3}{*}{ Acrílico } & Diâmetro médio das fibras & $\begin{array}{c}24 \mu \mathrm{m} \\
( \pm 4,59)\end{array}$ \\
\cline { 2 - 3 } & Gramatura $\left(\mathrm{g} / \mathrm{m}^{2}\right)$ & 600 \\
\cline { 2 - 3 } & Permeabilidade $\left(\mathrm{m}^{2}\right)$ & $1,7 \times 10^{-7}$ \\
\hline
\end{tabular}

\subsection{Unidade e Procedimento Experimental}

A unidade experimental está apresentada na Figura 2. Esta unidade consiste de um alimentador de partículas, um suporte para o meio filtrante, um sistema de limpeza por pulso de ar reverso, uma placa de orifício e um sistema de aquisição de dados.

O equipamento utilizado constituiu de uma área filtrante de $227 \mathrm{~cm}^{2}$. A filtração iniciava-se apenas quando a umidade dentro da caixa de alimentação atingia $20 \%$. A vazão mássica de pó alimentada no sistema foi de $0,015 \mathrm{~g} / \mathrm{s}$. Durante a filtração foi registrada a queda de pressão em função do tempo, por meio do sistema de aquisição de dados. Desta forma, todo experimento foi controlado por um software, fornecendo os registros das variáveis de interesse de operação. $\mathrm{O}$ valor de queda de pressão pré-estabelecido para cada ciclo de filtração foi de $200 \mathrm{~Pa}$ e a velocidade de filtração foi mantida constante em $4 \mathrm{~cm} / \mathrm{s}$. Durante a limpeza do filtro, utilizou-se o tempo de pulso de $200 \mathrm{~ms}$, para o desprendimento da camada de material particulado formada. O número de ciclos realizados para cada meio filtrante foi de 200 ciclos de filtração e limpeza. 


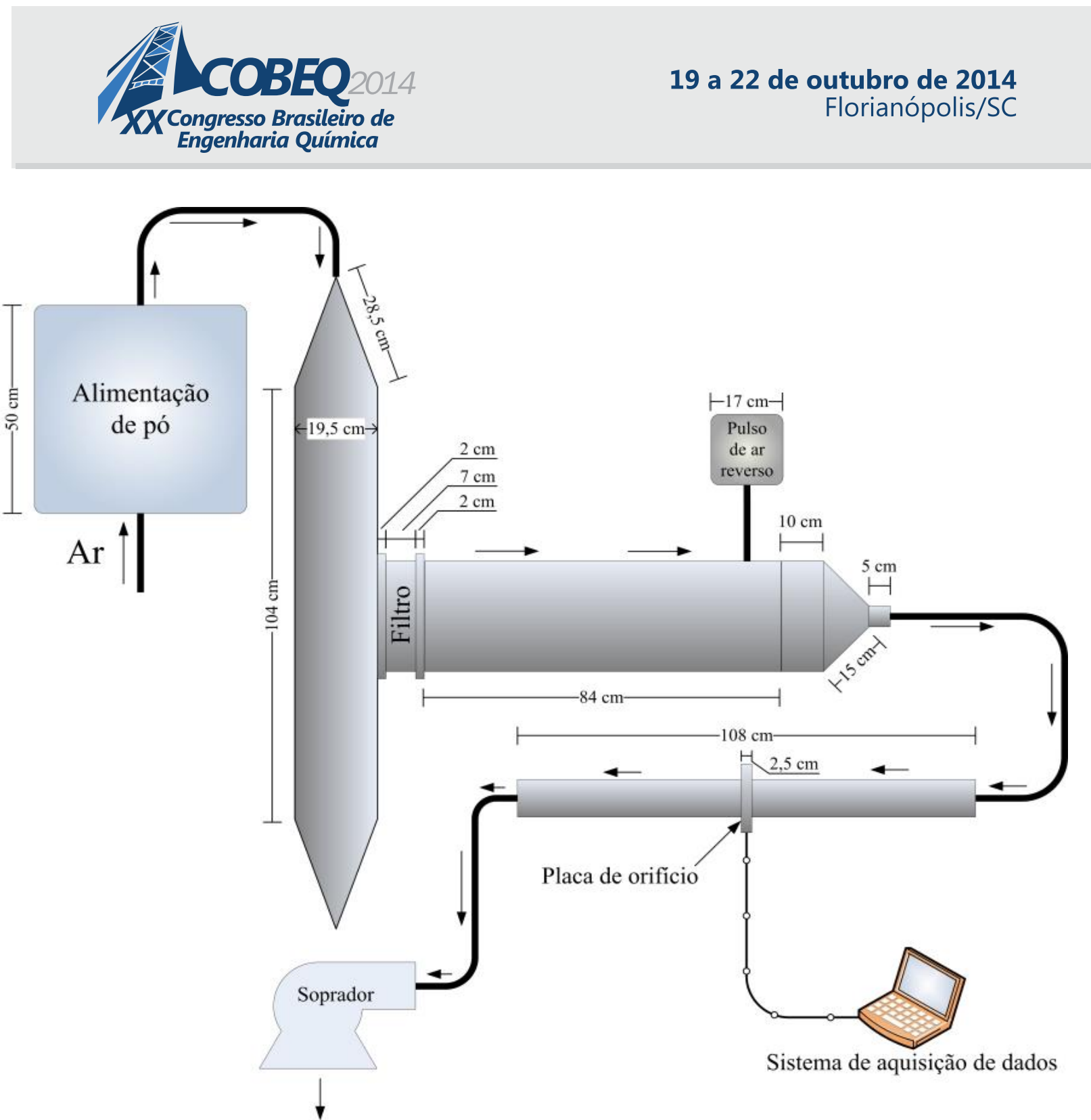

Figura 2 - Unidade Experimental.

\section{RESULTADOS E DISCUSSÕES}

Na Figura 3 são apresentados os resultados da queda de pressão em função do tempo de filtração durante os 200 ciclos de filtração por pulso de ar reverso. Ao analisar esta figura observou-se que os dois tecidos avaliados apresentaram tempos de filtração muito próximos, uma vez que os tempos de filtração foram 8663 e 8604 s, utilizando o feltro de polipropileno e o feltro de acrílico, respectivamente. Os comportamentos observados dos meios filtrantes foram provenientes devido às semelhanças nas estruturas das fibras, conforme apresentada na Tabela 1. Também na Figura 3, podese verificar que os dois filtros mostraram um comportamento típico de filtração de profundidade nos primeiros ciclos de filtração. Entretanto, após os 50 ciclos de filtração ficaram mais nítidos a ocorrência da filtração superficial. Isto se deve pelo fato da não remoção completa das partículas presentes internamente no meio filtrante, no qual resulta esta deposição mais superficial. 


\section{Acosese.}

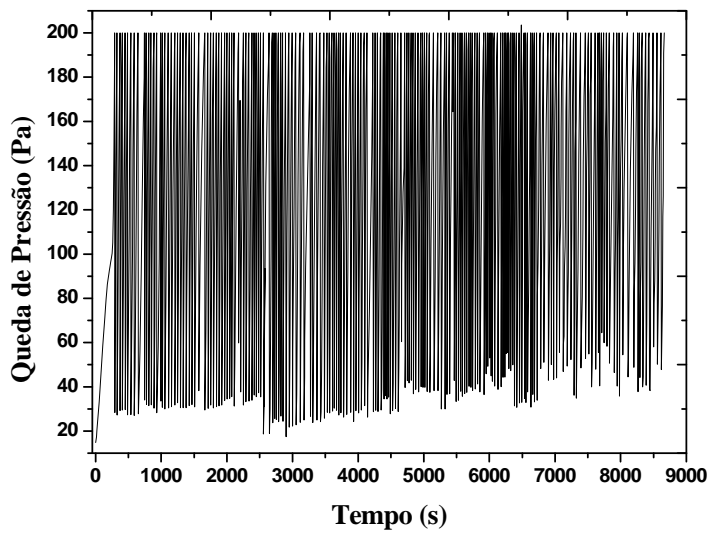

(a)
19 a 22 de outubro de 2014

Florianópolis/SC

Figura 3 - Ensaios de filtração e limpeza por pulso de ar reverso nos feltros:

(a) polipropileno e (b) acrílico.

Nas Figuras 4 e 5 são apresentados os resultados obtidos da queda de pressão residual e da massa retida por unidade de área em função do número de ciclos, respectivamente. A análise da Figura 4 mostrou que a queda de pressão residual aumentou com o número de ciclos. Isto é explicado devido ao aumento das partículas retidas no meio filtrante após a limpeza, conforme visualizado na Figura 5, proporcionando uma maior resistência à passagem do ar. Em relação aos meios filtrantes, os comportamentos de queda de pressão e massa retida foram bastante semelhantes.

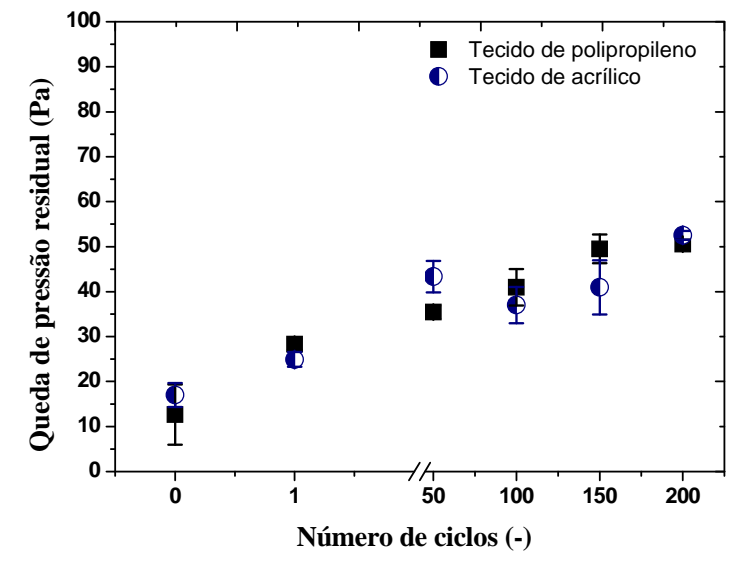

Figura 4: Resultados da queda de pressão residual em função do número de ciclos.

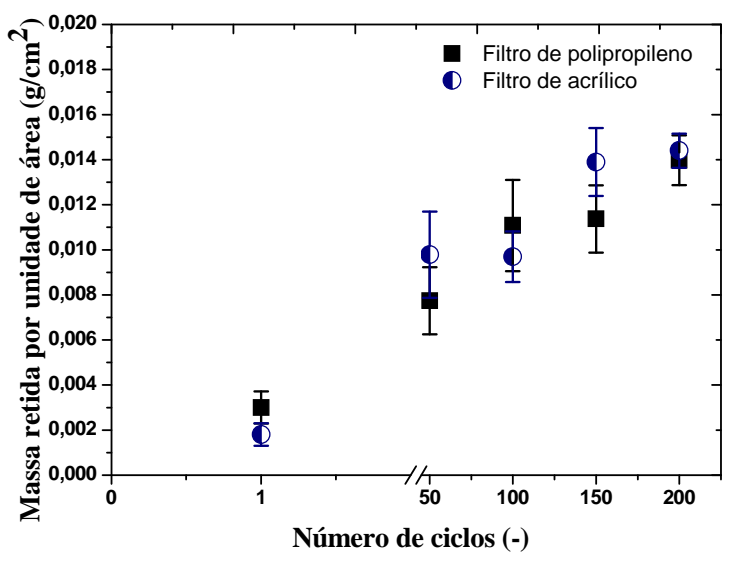

Figura 5: Resultados da massa retida por unidade de área em função do número de ciclos.

Na Figura 6 estão ilustradas as superfícies dos meios filtrantes após duzentos ciclos de filtração. Conforme esta Figura pode-se observar que o tecido de polipropileno apresentou maior quantidade de partículas na superfície em relação ao filtro de acrílico, bem como a maior permanência das partículas de concentrado fosfático preenchendo quase toda fibra do filtro. Por isso, no tecido de acrílico encontrou-se mais presente à filtração de profundidade, já que este apresentou maior permeabilidade, 


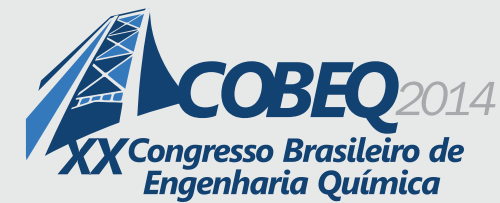

19 a 22 de outubro de 2014

Florianópolis/SC

menor quantidade de partículas retidas na superfície após a primeira limpeza do tecido por pulso de ar reverso.

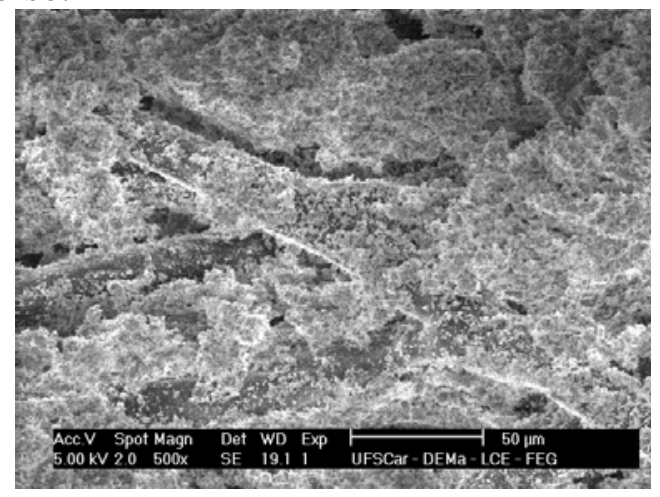

(a)

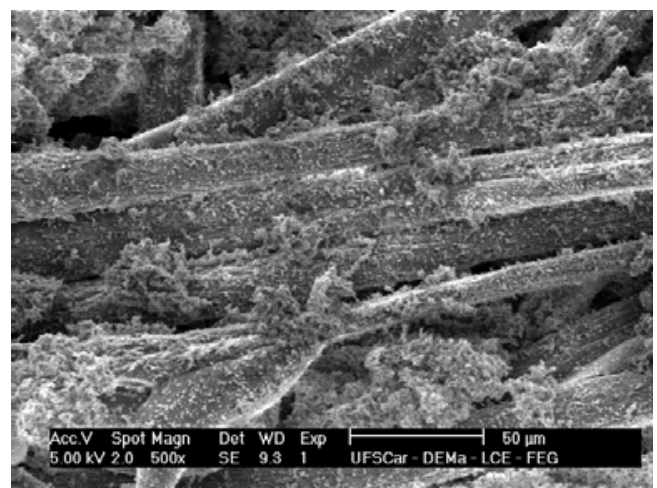

(b)

Figura 6 - Fotografias das superfícies dos tecidos de (a) polipropileno e de (b) acrílico gerados no MEV, após 200 ciclos de filtração por pulso de ar reverso, com ampliação de 500x.

Outra verificação importante na avaliação da regeneração dos filtros refere-se à fração de partículas removidas em cada limpeza do filtro. A fração de partículas removidas foi obtida, admitindo-se, a massa de pó alimentada constante, por meio da calibração do equipamento de alimentação de material particulado, durante a operação de filtração. Na Figura 7 estão apresentadas as frações de partículas removidas a cada limpeza em função do número de ciclos.

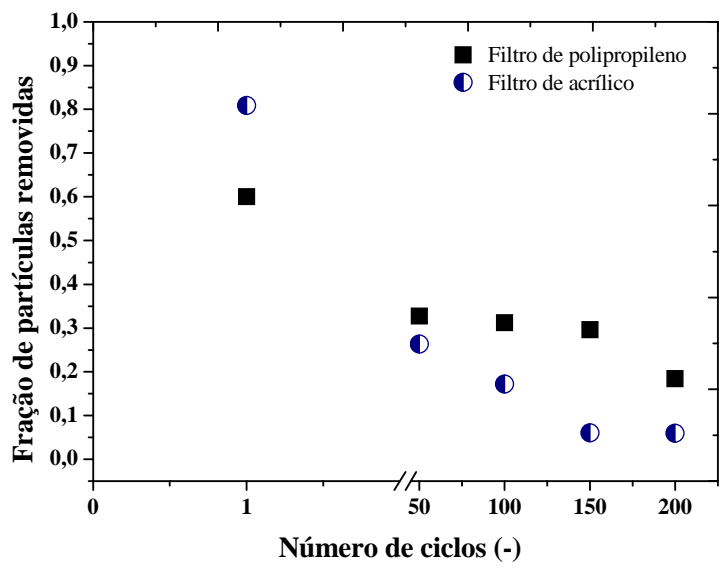

Figura 7 - Fração de massa removida da torta formada em função do número de ciclos.

Na Figura 7 pode-se constatar que, a fração removida após 200 ciclos de filtração e limpeza por pulso de ar reverso utilizando o tecido de polipropileno foi maior que utilizando o tecido de acrílico. Desta forma, o tecido de polipropileno apresentou maior capacidade de remoção de partículas utilizando a operação de remoção por pulso de ar reverso. Este comportamento pode ser explicado pelo fato do filtro de polipropileno apresentar uma filtração mais superficial em relação ao acrílico, que apresenta uma filtração mais de profundidade, o que facilitou a remoção da torta. Entretanto, ambos os meios filtrantes mostraram uma redução na eficiência de limpeza, utilizando os mesmos parâmetros operacionais. 
Através dos ensaios de filtração, foi possível obter a porosidade e a resistência específica da torta de filtração, conforme apresentada na Figura 8 e 9. A porosidade da torta de filtração $(\varepsilon)$ foi estimada a partir dos resultados de $\Delta P$ em função do $t$ e, utilizando a Equação de Ergun Modificada (SILVA et al.,1999):

$$
\frac{\Delta P}{t}=\frac{150 Q \mu V_{F}(1-\varepsilon)}{A_{C} \rho_{P} d_{P}^{2} \varepsilon^{3}}+\frac{1,75 Q \rho_{G} V_{F}^{2}}{A_{C} \rho_{P} d_{P}} \frac{1}{\varepsilon^{3}}
$$

em que $\Delta P$ representa a queda de pressão na direção do escoamento; $t$ o tempo de filtração; $Q$ a vazão mássica de pó; $\mu$ a viscosidade do fluido; $A_{C}$ a área superficial da torta de filtração; $\rho_{P}$ a densidade de partículas; $d_{P}$ o diâmetro médio das partículas.

Os resultados de resistência específica da torta foram determinados através da queda de pressão da torta pela velocidade superficial do gás $\left(\Delta P_{C} / V_{F}\right)$ em função da massa de material particulado depositado por unidade de área $(W)$ e, por meio de um ajuste linear, conforme indicado na equação a seguir (LEITH e ALLEN, 1986):

$$
\frac{\Delta P_{C}}{V_{F}}=k_{C} W
$$

sendo que $V_{F}$ é a velocidade superficial de filtração; $k_{C}$ é a resistência específica da torta; $W$ é a quantidade de material particulado depositado por unidade de área do meio filtrante e $\Delta P_{C}$ é a queda de pressões referentes a torta de filtração.

Na Figura 8 está apresentada a porosidade de torta teórica e na Figura 9 está apresentada a resistência específica de torta em relação ao número de ciclos.

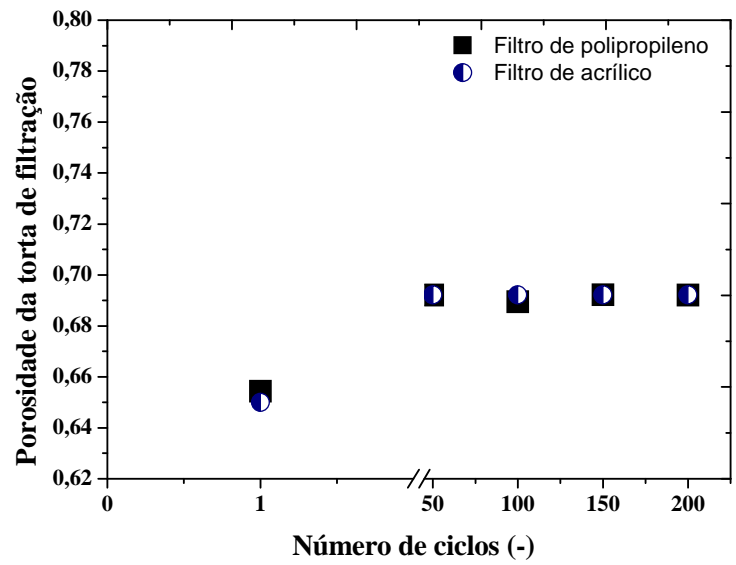

Figura 8: Porosidade teórica de torta de filtração em função o número de ciclos.

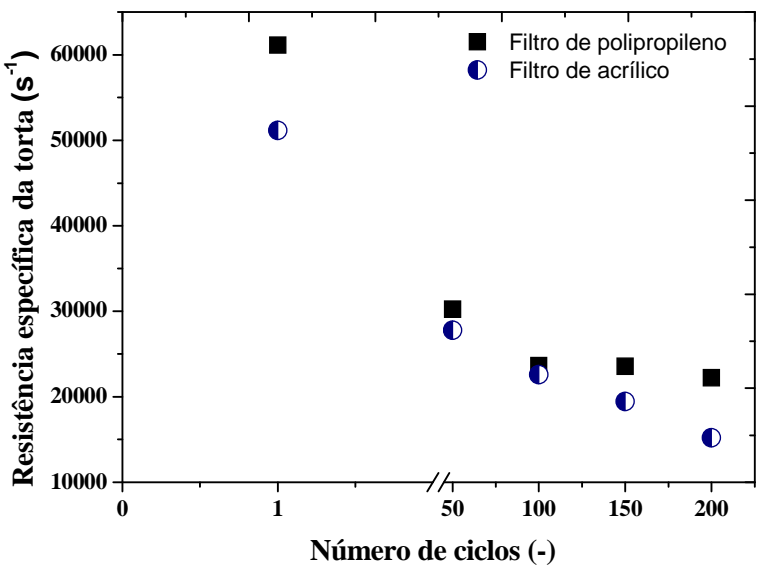

Figura 9: Resistência específica de torta em função do número de ciclos.

Através da Figura 8, observou-se que, as tortas foram menos porosas no primeiro ciclo de filtração, e posteriormente foram aumentando até o ducentésimo ciclo. Além disso, após os cinquenta 


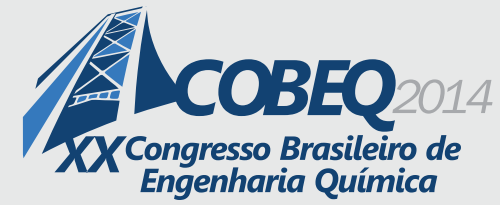

19 a 22 de outubro de 2014

Florianópolis/SC

ciclos de filtração notou-se que, manteve-se a porosidade da torta de filtração. A análise da Figura 9 constatou-se que, a resistência específica da torta tendeu a diminuir com o número de ciclos. Ainda de acordo com esta figura, após os duzentos ciclos de filtração e limpeza por pulso de ar reverso observou-se que o tecido de polipropileno apresentou maior resistência específica da torta em relação ao tecido de acrílico. Com isso, observou-se que, as tortas mais porosas apresentaram menor resistência específica da torta.

\section{CONCLUSÃO}

Os resultados apresentaram uma tendência de aumento da queda de pressão residual e da massa retida em função do número de ciclos, devido ao desgaste dos meios filtrantes, da retenção das partículas pelas fibras e a presença da saturação torta-tecido. Consequentemente, com o acréscimo do número de ciclos, observou-se o aumento da porosidade da torta de filtração, ocasionando uma maior eficiência de coleta durante a filtração. Entretanto, a resistência específica da torta diminuiu, assim como a fração de partículas removidas dos dois meios filtrantes avaliados. Assim, pode-se dizer que ambos os filtros apresentaram comportamentos de filtração semelhantes, provenientes das semelhanças nas estruturas das fibras.

\section{REFERÊNCIAS}

ALLEN, R. W. K.; GOYDER, H. G. D.; MORRIS, K. Modelling media movement during cleaning of pulse jet fabric filters. Trans IChemE, v.77, pat A, 1999.

GINESTET, A.; PUGNET, D. Measurement of the particle emission of an industrial pulse-jet fabric following filters cleaning. $11^{\text {th }}$ World Filtration Congress, Graz-Austria, 2012.

LEITH, D.; ALLEN, R.W.K. Dust filtration by Fabric Filters. Progress in Filtration and Separation, v.4, p.1-55, 1986.

MUKHOPADHYAY, A; CHOUDHARY, A.K. Performance of filter media as function of fibre fineness in pulse jet filtration system. Textiles and Light Industrial Science and Technology (TLIST) Volume 2 Issue 1, 2013.

PARK, B. H.; KIM, S. B.; JO, Y. M.; LEE, M-H. Filtration Characteristics of Fine Particulate Matters in a PTFE/Glass Composite Bag Filter. Aerosol and Air Quality Research, v.12, p. 1030-1036, 2012.

SALEEM, M.; KHAN, R. U.; TAHIR, M. S.; KRAMMER, G. Experimental study of cake formation on heat treated and membrane coated needle felts in a pilot scale pulse jet bag filter using optical insitu cake height measurement. Powder Technology, v.214, p. 388-399, 2011.

SILVA, C.R.N.; NEGRINI, V.S.; AGUIAR, M.L.; COURY, J.R. Influence of gas velocity on cake formation and detachment. Powder Technology, v.101, p.165-172, 1999.

SIMON, X; CHAZELET, S; THOMAS, D; BÉMER, D; RÉGNIER, R. Experimental study of pulsejet cleaning of bag filters supported by rigid rings. Powder Technology v.172, p.67-81, 2007.

SIMON, X.; BÉMER, D.; CHAZELET, S.; THOMAS, D.; RÉGNIER, R. Consequences of high transitory airflows generated by segmented pulse-jet cleaning of dust collector filter bags. Powder Technology v.201, p.37-48, 2010.

ZHOU, R.; SHEN, H.; ZHAO, M. Simulation studies on protector of pulse-jet cleaning filter bag. Energy Procedia, 16, p. 426-431, 2012. 